Proceedings

\title{
Joint Forces of HR-Spicp-MS and EAF4-MALS for Characterization of Gold Nanorods Conjugated with Synthetic Glycopolymers †
}

\author{
Milica Velimirovic 1,2, , Alessia Pancaro 2, Robert Mildner ${ }^{3}$, Panagiotis G. Georgiou ${ }^{4}$, \\ Kristof Tirez ${ }^{2}$, Inge Nelissen ${ }^{2}$, Christoph Johann ${ }^{3}$, Matthew I. Gibson ${ }^{4,5}$ and Frank Vanhaecke ${ }^{1}$ \\ 1 Atomic \& Mass Spectrometry-A\&MS Research Group, Department of Chemistry, Ghent University, \\ Campus Sterre, Krijgslaan 281-S12, 9000 Ghent, Belgium; frank.vanhaecke@ugent.be \\ 2 Flemish Institute for Technological Research (VITO), Boeretang 200, 2400 Mol, Belgium; \\ email1@gmail.com (A.P.); kristof.tirez@vito.be (K.T.); email2@gmail.com (I.N.) \\ 3 Wyatt Technology Europe GmbH, Hochstrasse 12a, 56307 Dernbach, Germany; \\ Robert.Mildner@wyatt.eu (R.M.); email3@gmail.com (C.J.) \\ 4 Department of Chemistry, University of Warwick, Gibbet Hill Road, Coventry CV4 7AL, UK; \\ email1@gmail.com (P.G.G.); M.I.Gibson@warwick.ac.uk (M.I.G.) \\ 5 Warwick Medical School, University of Warwick, Gibbet Hill Road, Coventry CV4 7AL, UK \\ * Correspondence: milica.velimirovic@vito.be; Tel.: +32-14-335779 \\ + Presented at the 2nd International Online-Conference on Nanomaterials, 15-30 November 2020; \\ Available online: https://iocn2020.sciforum.net/.
}

Published: 15 November 2020

\begin{abstract}
As the diversity of nanomaterials is wide and their size can vary by 2 orders of magnitude (1-100 nm), the development of new and advanced analytical tools for their in-depth characterization is of paramount importance, allowing a fundamental understanding of their structure, further alteration and degree of chemical surface functionalization. Herein, we present a new strategy for characterization of gold nanorods (GNRs) that are of specific interest for biomedical applications due to their unique size-dependent longitudinal surface plasmon resonance band in the visible to near-infrared spectral region. More precisely, we characterized GNRs conjugated with short and long synthetic glycopolymers for biosensing of lectins in terms of particle size, coating thickness, and/or mobility properties in comparison with the bare GNRs. This endeavour requires a multidisciplinary approach including a new comprehensive set of fit-for-purpose analytical tools being high-resolution single-particle inductively coupled plasma-mass spectrometry (HR-spICPMS) and electrical asymmetric-flow field-flow-fractionation hyphenated to a multi-angle light scattering detector (EAF4-MALS). GNRs were separated and characterized via EAF4-MALS in terms of their size and charge, while HR-spICP-MS provided information on the particle number density, size, size distribution, and the dimensional characterisation. In addition, EAF4-MALS appears to be suitable for estimating coating thickness of glycoconjugated GNRs.
\end{abstract}

Keywords: gold nanorods; high-resolution single-particle inductively coupled plasma-mass spectrometry; electrical asymmetric-flow field-flow-fractionation hyphenated to a multi-angle light scattering detector

\section{Introduction}

Nanomaterials have attracted considerable attention because of their unique physical, chemical, and mechanical properties, exhibiting distinct size-dependent properties in the 1-100 $\mathrm{nm}$ range. Intensive research is being done related to the use of nanomaterials in many contexts such as energy 
storage, energy conversion, solar cells, pharmaceuticals, life science applications, optoelectronics, sensing and actuation nanosystems, catalysis, and composite materials [1]. Since the potential of nanoscale particles (NPs) has been identified, scientific and commercial interest has increased tremendously. It is expected that in the next decade, a countless number of increasingly complex, versatile, and useful nanomaterials will be designed, synthesized, and applied in different research areas. However, these advanced applications of nanomaterials require their customization in terms of chemical composition/functionalization, size, and morphology. Careful and safe design of NPs and precise control during the synthesis process to ensure a successful application is crucial [2]. As a result, development of different analytical tools to follow-up the nanomaterial synthesis from an early stage onwards on the one hand and to fully characterize the finally synthesized NPs on the other is required.

Gold nanorods (GNRs) have been found promising in biomedical applications, mainly because of their unique optical properties dominated by the localized surface plasmon resonance (LSPR) phenomenon: their anisotropic shape causes a splitting of their optical absorption bands into two peaks corresponding to the transverse and longitudinal plasmon resonances. The longitudinal resonance peak position is highly shape- and size dependent, and it is shifted from the visible to the near-infrared (NIR) region with increasing aspect ratio (length/width) [3,4]. GNRs are promising in cancer diagnostics [5] and treatment [6-11], gene therapy and drug deliver applications [12,13], as well as for in vitro diagnostics of a wide variety of disease-specific biomarker targets [14-16].

The utilization of GNRs for biomedical applications requires an appropriate functionalization to provide chemical stability and biocompatibility, and to recognize target molecules in a biological environment. However, the synthesis and characterization of GNRs with well-defined sizes, shapes, and bioconjugated surfaces remains an important challenge.

A unique aspect of this specific research is the development of a set of analytical tools (methods) for physicochemical characterization of gold nanorods (GNRs) conjugated with short and long synthetic glycopolymers for biosensing of lectins in terms of particle size, coating thickness, and/or mobility properties in comparison with the bare GNRs. The developmental process of glycoconjugated GNRs requires a multidisciplinary analytical approach including a new comprehensive set of fit-for-purpose tools, such as high-resolution single-particle inductively coupled plasma-mass spectrometry (HR-spICP-MS) and electrical asymmetric-flow field-flowfractionation hyphenated to a multi-angle light scattering detector (EAF4-MALS).

\section{Materials and Methods}

\subsection{Glycopolymer Conjugated Gold Nanorods}

Citrate-stabilized GNRs of $10 \mathrm{~nm}$ width and $38 \mathrm{~nm}$ length were purchased from Nanopartz (Loveland CO US/Canada). Monomer N-hydroxyethyl acrylamide (97\%, HEA), 2(dodecylthiocarbonothioylthio)-2-methylpropionic acid pentafluorophenyl ester (98\%, PFP-DMP), and D-(+)-galactosamine were all purchased from Sigma-Aldrich. Poly(N-hydroxyethyl acrylamide) (PHEA) was synthetized by photo-initiated reversible addition-fragmentation chain transfer (RAFT) polymerization in two lengths of 35 and 60 , corresponding to different degrees of polymerization; then they were functionalized with D-(+)-galactosamine (Gal) achieving the glycopolymers GalPHEA35 and Gal-PHEA60, as previously described [17].

Citrate-GNRs were functionalized with $4 \mathrm{mg}$ of each glycopolymer dissolved in $200 \mu \mathrm{L}$ of water and mixed by pipetting with $800 \mu \mathrm{L}$ of GNRs suspension at $10 \mathrm{OD}$. After $1 \mathrm{~h}$ incubation at room temperature in the dark, the particles were sonicated for 1 min using an ultrasonic bath at $40 \mathrm{kHz}$ (Branson 1800 series CPX1800H), centrifuged at $12000 \mathrm{RCF}$ and $20{ }^{\circ} \mathrm{C}$ for 15 min using a Sigma 330KS centrifuge, and the supernatant removed. This was followed by 3 cycles of re-suspension in 1 $\mathrm{mL}$ water, centrifugation and decanting. The particles were finally resuspended in $1 \mathrm{~mL}$ water and stored in polypropylene graduated tubes at $4{ }^{\circ} \mathrm{C}$ until use.

\subsection{HR-Spicp-MS}


All samples were analyzed with a Nu Attom ICP-MS (Nu Instruments Ltd., Wrexham, UK). This instrument is equipped with a double-focusing sector field mass spectrometer, with forward (NierJohnson) geometry. In single particle mode, a single $\mathrm{m} / \mathrm{z}$ value was monitored (i.e., the magnetic field and all voltages were fixed). For the measurement of GNRs the instrument was operated at low resolution $(\mathrm{R} \sim 300)$. Samples were introduced using a conventional sample introduction system, consisting of a glass concentric nebulizer mounted onto a quartz cyclonic spray chamber with a

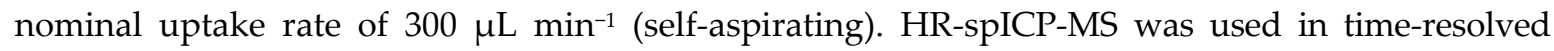
analysis (TRA) measurement mode, with dwell times of $40 \mu$ s and a total acquisition time of $60 \mathrm{~s}$ (Figure 1, Table 1).

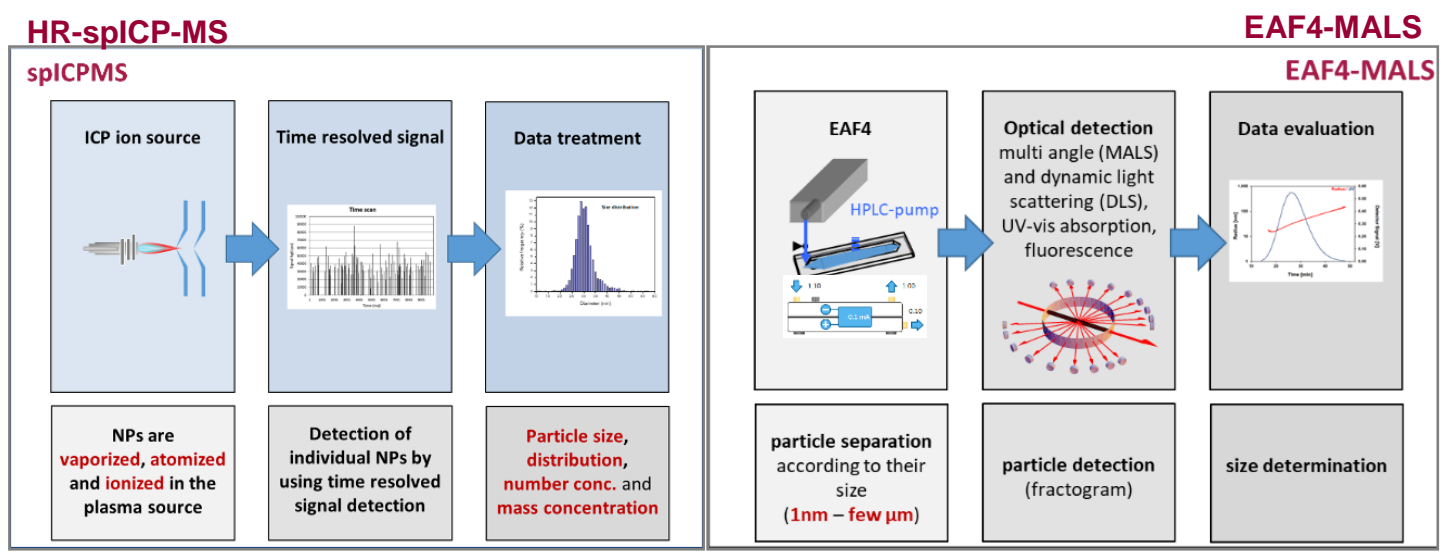

Figure 1. HR-spICP-MS and EAF4-MALS set up.

Table 1. ICP-MS instrumental settings and conditions.

\begin{tabular}{cc}
\hline Parameter & \\
\hline RF power & $1300 \mathrm{~W}$ \\
Plasma gas flow rate & $13 \mathrm{~L} \mathrm{~min}^{-1}$ \\
Carrier gas flow rate & $0.93 \mathrm{~L} \mathrm{~min}^{-1}$ \\
Measurement mode & $\mathrm{TRA}$ \\
Nuclide monitored & ${ }^{197} \mathrm{Au}$ \\
Dwell time & $40 \mu \mathrm{s}$ \\
Acquisition time & $60 \mathrm{~s}$ \\
Nebulizer & MicroMist \\
Spray chamber & Cyclonic \\
\hline
\end{tabular}

Data acquisition and data treatment were performed using the combination of NuAttoLab and NuQuant sofware ( $\mathrm{Nu}$ Instruments, Ltd., Wrexham, UK). Finally, GNRs dimensional characterization method was adopted from Kalomista et al., 2017 [18].

\subsection{EAF4-MALS}

EAF4 measurements were performed using an Eclipse AF4 system with Mobility module (Wyatt Technology, Santa Barbara, CA, USA) using a $25 \mathrm{~cm}$ Mobility electric long channel equipped with a $350 \mu \mathrm{m}$ spacer and a $5 \mathrm{kDa}$ cutoff polyether sulfone membrane [19]. Detection was accomplished with a DAWN 18-angle MALS detector (Wyatt Technology, Santa Barbara, CA, USA). $0.5 \mathrm{mM}$ sodium nitrate was used as the carrier solution. For separation, the cross-flow was maintained at $0.5 \mathrm{~mL} / \mathrm{min}$ for $30 \mathrm{~min}$. For Mobility measurements an amperage of $-0.1 \mathrm{~mA}$ (bottom electrode positively charged) was applied during separation. Data were collected and analyzed using VISION software (Wyatt Technology, Santa Barbara, CA, USA). Effective channel height calibration was performed using $20 \mathrm{~nm}$ spherical gold nanoparticles (BAM-N004, Bundesanstalt für Materialforschung und prüfung, Berlin, Germany). 


\section{Results}

\subsection{HR-spICP-MS for Glycoconjugated GNRs Characterization}

High temporal resolution extends the capabilities of the spICP-MS technique as previously reported by Kalomista et al. [18]. As expected, the average of $5 \%$ for shortest and longest transit times were correlated with the length of $32 \mathrm{~nm}$ and the width of $11 \mathrm{~nm}$ of GNRs, respectively. This is in accordance with the certificates provided for GNRs (10 nm width and $38 \mathrm{~nm}$ length). As such HRspICP-MS allowed fast dimensional characterization of GNRs and could be used as an orthogonal method to investigate different batches of the GNRs after synthesis and their further use in conjugation with short and long synthetic glycopolymers.

The spherical equivalent diameter and particle size distribution data for bare GNRs and glycoconjugated GNRs (Figure 2, Table 2) was derived in order to provide the thickness of the glycopolymers binded on the GNRs surface. When interpreting mean particle size and particle size distribution data result as provided by HR-spICP-MS, it must be acknowledged that the particle size was not significantly altered after binding synthetic glycopolymers on the GNRs surface, meaning that both short and long glycopolymers did not impact the particle size measurements. This is an important factor as the localized surface plasmon resonance signal generated by GNRs is shape- and size-dependent $[5,6]$. However, HR-sp-ICP-MS is not able to provide information on the thickness of the glycopolymers bound to the GNR surface as it is basedon single particle detection via the ${ }^{197} \mathrm{Au}$ ion signal. Therefore, the EAF4-MALS method was further applied.

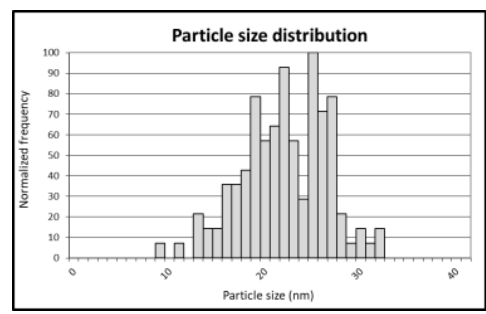

(a)

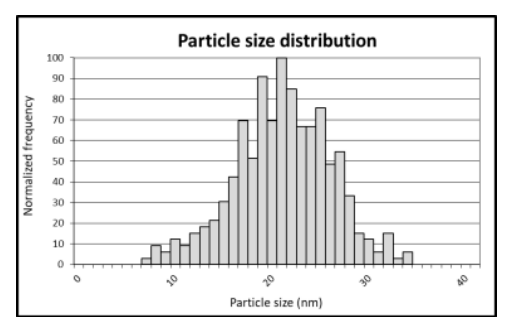

(b)

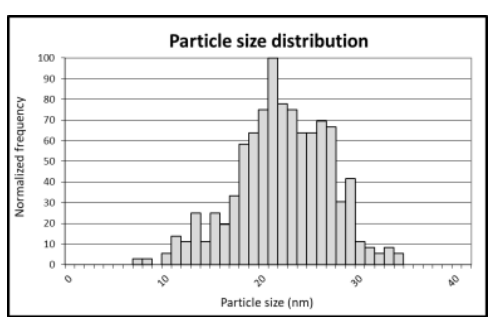

(c)

Figure 2. Particle size distribution of (a) bare GNRs; (b) GNR-Gal-PHEA35; and (c) GNR-GalPHEA60.

Table 2. HR-spICP-MS results of bare GNRs and glycoconjugated GNRs.

\begin{tabular}{cccc}
\hline & GNRs & GNR-Gal-PHEA35 & GNR-Gal-PHEA60 \\
\hline Spherical equivalent diameter $(\mathrm{nm})$ & $21.0 \pm 0.5$ & $21.0 \pm 0.4$ & $22.0 \pm 0.0$ \\
\hline
\end{tabular}

\subsection{EAF4-MALS for Glycoconjugated GNRs Characterization}

The size of GNRs was assessed using EAF4-MALS runs without an amperage applied during separation (Figure 3).

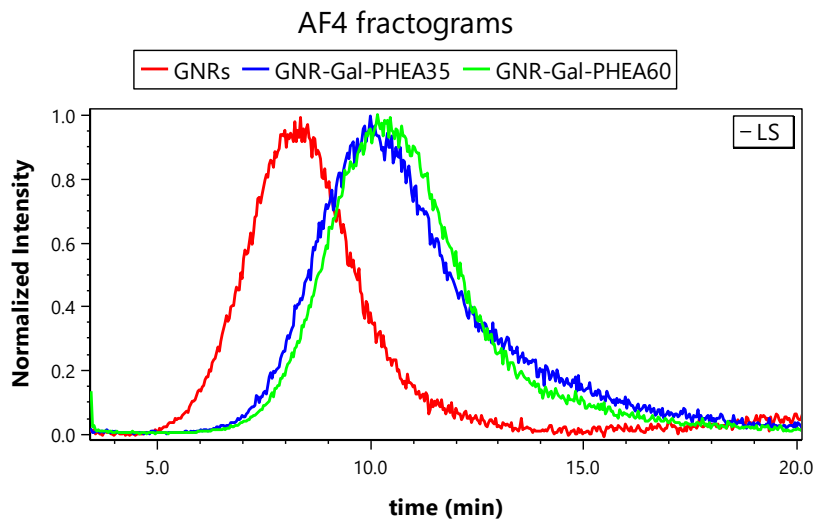


Figure 3. $90^{\circ}$ light scattering fractograms of GNRs, GNR-Gal-PHEA35 and GNR-Gal-PHEA60.

The retention time could then be directly related to the sample hydrodynamic size. We obserevd an increased retention time of glycoconjugated GNRs compared to the bare GNRs (Figure 3) indicating an increase in hydrodynamic size which was highest for the longest glycopolymers. In addition, HR-spICP-MS results have shown that there was no significant difference in spherical equivalent diameter of the bare GNRs and glycoconjugated GNRs. Thereofre, the increase in hydrodynamic diameter can be attributed to the glycoconjugation of GNRs. This confirmed successful coating of GNRs. The increase in size (Table 3) can be used to estimate the coating thickness of glycoconjugated GNRs. The charge of GNRs is assessed by comparing EAF4-MALS runs with (-0.1 $\mathrm{mA})$ and without $(0.0 \mathrm{~mA})$ an amperage applied during separation. Shifts towards a higher retention time of up to $0.5 \mathrm{~min}$ are observed for each sample when applying $-0.1 \mathrm{~mA}$. This indicates a negative charge of GNRs before and after glycoconjugation.

Table 3. Hydrodynamic diameter Dh determined via EAF4-MALS.

\begin{tabular}{cccc}
\hline & GNRs & GNR-Gal-PHEA35 & GNR-Gal-PHEA60 \\
\hline $\mathrm{D}_{\mathrm{h}}(\mathrm{nm})$ & 17.0 & 21.3 & 22.1 \\
\hline
\end{tabular}

\section{Conclusions}

The current study achieved its main goals concerning the characterization of the GNRs conjugated with short and long synthetic glycopolymers. The GNRs were separated and characterized via EAF4-MALS regarding size and surface charge, while HR-spICP-MS provided information on the particle number density, size, size distribution, and anisotropic dimensions. In addition, EAF4-MALS appeared to be suitable for estimating the coating thickness of glycoconjugated GNRs. Finally, further research is needed to prove the significant advantage offered by joining the capabilities of HR-spICP-MS and EAF4-MALS (e.g., reproducibility) for the characterization of gold nanorods conjugated with synthetic glycopolymers when compared to more common characterization methods, such as UV-VIS and DLS.

Acknowledgments: Milica Velimirovic is a senior postdoctoral researcher of the Research FoundationFlanders (FWO project number 12ZD120N). Alessia Pancaro, Panagiotis G. Georgiou, Inge Nelissen and Matthew I. Gibson thank the European Union's H2020-MSCA-ITN programme under Grant Agreement $\mathrm{N}^{\circ}$ 814236 (NanoCarb).

\section{References}

1. Curtis, A.S.G.; Wilkinson, C. Nantotechniques and approaches in biotechnology. Trends Biotech. 2001, 19, 97.

2. Mülhopt, S.; Diabaté, S.; Dilger, M.; Adelhelm, C.; Anderlohr, C.; Bergfeldt, T.; de la Torre, J.G.; Jiang, Y.; Valsami-Jones, E.; Langevin, D.; et al. Characterization of Nanoparticle Batch-To-Batch Variability. Nanomaterials 2013, 8, 311.

3. Smith, M.; Mancini, M.C.; Nie, S.M. BIOIMAGING second window for in vivo imaging. Nat. Nanotechnol. 2009, 4, 710 .

4. Zhu, K.; Yong, T.; Roy, I.; Hu, R.; Ding, H.; Zhao, L.L.; Swihart, M.T.; He, G.S.; Cui, Y.P.; Prasad, P.N. Additive controlled synthesis of gold nanorods (GNRs) for two-photon luminescence imaging of cancer cells. Nanotechnology 2010, 21, 285106.

5. Li, J.L.; Gu, M. Surface plasmonic gold nanorods for enhanced two-photon microscopic imaging and apoptosis induction of cancer cells. Biomaterials 2010, 31, 9492.

6. Dickerson, E.B.; Dreaden, E.C.; Huang, X.H.; El-Sayed, I.H.; Chu, H.H.; Pushpanketh, S.; McDonald, J.F.; El-Sayed, M.A. Gold nanorod assisted near-infrared plasmonic photothermal therapy (PPTT) of squamous cell carcinoma in mice. Cancer Lett. 2008, 269, 57.

7. XHuang, H.; El-Sayed, I.H.; Qian, W.; El-Sayed, M.A. Cancer cell imaging and photothermal therapy in the near-infrared region by using gold nanorods. J. Am. Chem. Soc. 2006, 128, 2115. 
8. Okuno, T.; Kato, S.; Hatakeyama, Y.; Okajima, J.; Maruyama, S.; Sakamoto, M.; Mori, S.; Kodama, T. Photothermal therapy of tumors in lymph nodes using gold nanorods and near-infrared laser light. J. Control. Release 2013, 172, 879.

9. Kuo, W.S.; Chang, C.N.; Chang, Y.T.; Yang, M.H.; Chien, Y.H.; Chen, S.J.; Yeh, C.S. Gold nanorods in photodynamic therapy, as hyperthermia agents, and in near-infrared optical imaging. Angew. Chem. Int. Ed. 2010, 49, 2711.

10. Li, L.; Chen, J.Y.; Wu, X.; Wang, P.N.; Peng, Q.A. Plasmonic gold nanorods can carry sulfonated aluminum phthalocyanine to improve photodynamic detection and therapy of cancers. J. Phys. Chem. B 2010, 114, 17194.

11. Chen, C.C.; Lin, Y.P.; Wang, C.W.; Tzeng, H.C.; Wu, C.H.; Chen, Y.C.; Chen, C.P.; Chen, L.C.; Wu, Y.C. DNA-gold nanorod conjugates for remote control of localized gene expression by near infrared irradiation. J. Am. Chem. Soc. 2006, 128, 3709.

12. Huang, H.C.; Barua, S.; Kay, D.B.; Rege, K. Simultaneous enhancement of photothermal stability and gene delivery efficacy of gold nanorods using polyelectrolytes. ACS Nano 2009, 3, 2941.

13. Nusz, G.J.; Curry, A.C.; Marinakos, S.M.; Wax, A.; Chilkoti, A. Rational selection of gold nanorod geometry for label-free plasmonic biosensors. ACS Nano. 2009, 3, 795-806. doi:10.1021/nn8006465.

14. Guo, Y.J.; Sun, G.M.; Zhang, L.; Tang, Y.J.; Luo, J.J.; Yang, P.H. Multifunctional optical probe based on gold nanorods for detection and identification of cancer cells. Sens. Actuator B Chem. 2014, 191, 741.

15. Truong, P.L.; Cao, C.; Park, S.; Kim, M.; Sim, S.J. A new method for non-labeling attomolar detection of diseases based on an individual gold nanorod immunosensor. Lab Chip. 2011, 11, 2591.

16. Georgiou, P.G.; Baker, A.N.; Richards, S.J.; Laezza, A.; Walker, M.; Gibson, M. Tuning aggregative versus non-Aggregative lectin binding with glycosylated nanoparticles by the nature of the polymer ligand. J. Mater. Chem. B 2019, 8, 136.

17. Kálomista, I.; Kéri, A.; Ungor, D.; Csapó, E.; Dékány, I.; Prohaska, T.; Galbács, G. Dimensional characterization of gold nanorods by combining millisecond and microsecond temporal resolution single particle ICP-MS measurements J. Anal. At. Spectrom. 2017, 32, 2455.

18. Johann, C. WP2606: Exosome Characterization with FFF-MALS-DLS; Wyatt: 2018. 